\title{
Special issue: Invited papers from the symposium "Zoological Endeavours Inspired by A. Richard Palmer" held during the Annual Meeting of the Canadian Society of Zoologists (University of Windsor, 14-16 May 2019)
}

\author{
Guest Editors \\ Tetsuto Miyashita, Canadian Museum of Nature, P.O. Box 3443, Station D, Ottawa, ON K1P 6P4, Canada \\ Heather A. Jamniczky, Department of Cell Biology and Anatomy, Cumming School of Medicine, University of Calgary, \\ Calgary, AB T2X 4N1, Canada \\ Emily M. Standen, Department of Biology, University of Ottawa, Ottawa, ON K1N 6N5, Canada
}

\section{Symposium Organizers}

Tetsuto Miyashita, Canadian Museum of Nature, P.O. Box 3443, Station D, Ottawa, ON K1P 6P4, Canada Marjan Barazandeh, Faculty of Pharmaceutical Sciences, The University of British Columbia, 2405 Wesbrook Mall, Vancouver, BC V6T 1Z3, Canada

Christopher B. Cameron, Département de sciences biologiques, Université de Montréal, 1375, avenue Thérèse-Lavoie-Roux, Montréal, QC H2V 0B3, Canada 